\title{
Visit-to-visit variability of fasting plasma glucose as predictor of ischemic stroke: competing risk analysis in a national cohort of Taiwan Diabetes Study
}

Cheng-Chieh Lin ${ }^{1,2,3+}$, Chun-Pai Yang ${ }^{4,5,6+}$, Chia-Ing Li $i^{2,3}$, Chiu-Shong Liu' ${ }^{1,2,3}$, Ching-Chu Chen ${ }^{7,8}$, Wen-Yuan Lin ${ }^{1,3}$, Kai-Lin Hwang ${ }^{9}$, Sing-Yu Yang ${ }^{10}$ and Tsai-Chung Li $i^{10,11^{*}}$

\begin{abstract}
Background: Glycemic variation as an independent predictor of ischemic stroke in type 2 diabetic patients remains unclear. This study examined visit-to-visit variations in fasting plasma glucose (FPG), as represented by the coefficient of variation (CV), for predicting ischemic stroke independently, regardless of glycated hemoglobin $\left(\mathrm{HbA}_{1} \mathrm{c}\right)$ and other conventional risk factors in such patients.

Methods: Type 2 diabetic patients enrolled in the National Diabetes Care Management Program, $\geq 30$ years old and free of ischemic stroke $(n=28,354)$ in 2002 to 2004 were included, and related factors were analyzed with extended Cox proportional hazards regression models of competing risk data on stroke incidence.

Results: After an average 7.5 years of follow-up, there were 2,250 incident cases of ischemic stroke, giving a crude incidence rate of 10.56/1,000 person-years (11.64 for men, 9.63 for women). After multivariate adjustment, hazard ratios for the second, third and fourth versus first FPG-CV quartile were $1.11(0.98,1.25), 1.22(1.08,1.38)$ and 1.27 $(1.12,1.43)$, respectively, without considering $\mathrm{HbA}_{1} \mathrm{c}$, and $1.09(0.96,1.23), 1.16(1.03,1.31)$ and $1.17(1.03,1.32)$, respectively, after considering $\mathrm{HbA}_{1} \mathrm{c}$.
\end{abstract}

Conclusions: Besides $\mathrm{HbA}_{1} \mathrm{C}, \mathrm{FPG}-\mathrm{CV}$ was a potent predictor of ischemic stroke in type 2 diabetic patients, suggesting that different therapeutic strategies now in use be rated for their potential to (1) minimize glucose fluctuations and (2) reduce $\mathrm{HbA}_{1} \mathrm{c}$ level in type 2 diabetic patients to prevent ischemic stroke.

Keywords: Glucose variation, Ischemic stroke, Type 2 diabetes

\section{Background}

Diabetes ranks as a leading cause of incident ischemic stroke [1-3]. Epidemiological study confirms that diabetes independently raises the risk of ischemic stroke, the relative risk ranging from 1.8 - to nearly 6 -fold [3]. A glycated hemoglobin $\left(\mathrm{HbA}_{1} \mathrm{c}\right)$ level of $<7.0 \%$ is recommended by the American Diabetes Association (ADA) to prevent microvascular complications in type 2 diabetes

\footnotetext{
* Correspondence: tcli@mail.cmu.edu.tw

${ }^{\dagger}$ Equal contributors

${ }^{10}$ Graduate Institute of Biostatistics, College of Management, China Medical

University, Taichung, Taiwan

${ }^{11}$ Department of Healthcare Administration, College of Medical and Health Science, Asia University, Taichung, Taiwan

Full list of author information is available at the end of the article
}

patients $[4,5]$. Whether such control reduces risk of cardiovascular events and stroke remains unclear [5]. This has prompted large-scale studies to gauge macrovascular complications (including ischemic stroke) in such cases. Recent attention has targeted oscillating glucose levels possibly superimposed on $\mathrm{HbA}_{1} \mathrm{c}$ in affecting risks of complications [6-8]. Both in vitro and animal studies confirm that oscillating plasma glucose has a greater effect on endothelial function and oxidative-stress generation than constant high glucose $[9,10]$. Human studies correlate coefficient of variation (CV) of fasting plasma glucose (FPG) to outcome in type 2 diabetic patients, focusing on all-cause or cause-specific mortality [11-15]. Glycemic variation, determined by FPG-CV, as an independent 
predictor of ischemic stroke in type 2 diabetic patients remains unclear. Our study examined whether FPG variation, as measured by $\mathrm{CV}$, showed a significant independent clinical association with ischemic stroke, regardless of $\mathrm{HbA}_{1} \mathrm{c}$ and other conventional risk factors.

\section{Methods}

\section{Study population}

This retrospective cohort, encompassing all enrollees in a National Diabetes Care Management Program (NDCMP) in Taiwan, is a population-based study of 63,084 ethnic Chinese type 2 diabetic patients enrolled in the NDCMP in Taiwan during 2002 to 2004. Date of entry into NDCMP was defined as the index date. NDCMP is a case management program established by the National Health Insurance (NHI) Bureau in 2002. Those with clinically diagnosed diabetes based on ADA criteria (International Classification of Disease, 9th Revision, Clinical Modification (ICD-9- CM) diagnosis code 250) were recruited without restriction for anti-diabetes medication. Type 2 diabetic patients treated with diverse insulin sensitizers, insulin secretagogues and insulin regimens were included. For diagnosis of a new patient, an individual with FPG $>126 \mathrm{mg} / \mathrm{dl}$ (or $7.0 \mathrm{mmol} / \mathrm{L}$ ) or plasma glucose $\geq 200 \mathrm{mg} / \mathrm{dl}$ (or $11.1 \mathrm{mmol} / \mathrm{L}$ ) during an oral glucose tolerance test (OGTT) repeats the test on a different day to increase the validity of the diabetes diagnosis. Exclusion criteria were type 1 diabetes (ICD-9-CM code 250.x1/x3), gestational diabetes (ICD-9-CM code 648.83) and stroke (ICD-9-CM code 430-438), as well as being younger than 30 years of age. We enrolled patients with more than two recorded follow-ups of at least one year to rate FPG variability; 31,689 were eligible. Excluding those with missing data left 28,354 for analysis (Figure 1). We compared baseline characteristics between the patients included and those excluded using standardized mean differences. All standardized mean differences were less than 0.1 standard deviations (SD), indicating a negligible difference in means or proportions between groups. The study was approved by the China Medical University Hospital Ethical Review Board.

\section{Data sources for baseline and follow-up assessments}

As of March 1995, Taiwan's government launched an NHI program that covered approximately $99 \%$ of its population of 23.74 million in 1999 [16]. By the end of 2010, NHI contracted with $100 \%$ of hospitals and $92 \%$ of clinics island-wide [17]. The database includes patient demographics, diagnoses, and prescriptions in hospital and outpatient claims; claims data are randomly audited by the NHI Bureau. Expert reviews on a random sample for every 50 to 100 ambulatory and inpatient claims in each hospital and clinic are conducted quarterly to enhance the validity of claim data, and a severe penalty is imposed for a false diagnostic report by the NHI Bureau. This study used datasets for inpatient care by admission and outpatient visits during 2002 to 2010. Individuals in Taiwan carry unique personal identification numbers (PIN). For security and privacy purposes, patient identity data is scrambled cryptographically by the National Health Insurance Research Database (NHIRD). All NHI datasets can be interlinked with the PIN of each individual. Data comprise information for all insured subjects with regard to demographic data, date and source of diagnosis, ambulatory care, inpatient admission and outpatient/inpatient treatment. ICD-9-CM codes were used to identify individual health status. Due to comprehensive coverage of the NHI program, the proportion of enrollees withdrawing from $\mathrm{NHI}$ is very low; thus, bias due to being lost to follow-up is negligible. Enrollees underwent comprehensive assessment of their disease status and complications as well as a series of blood

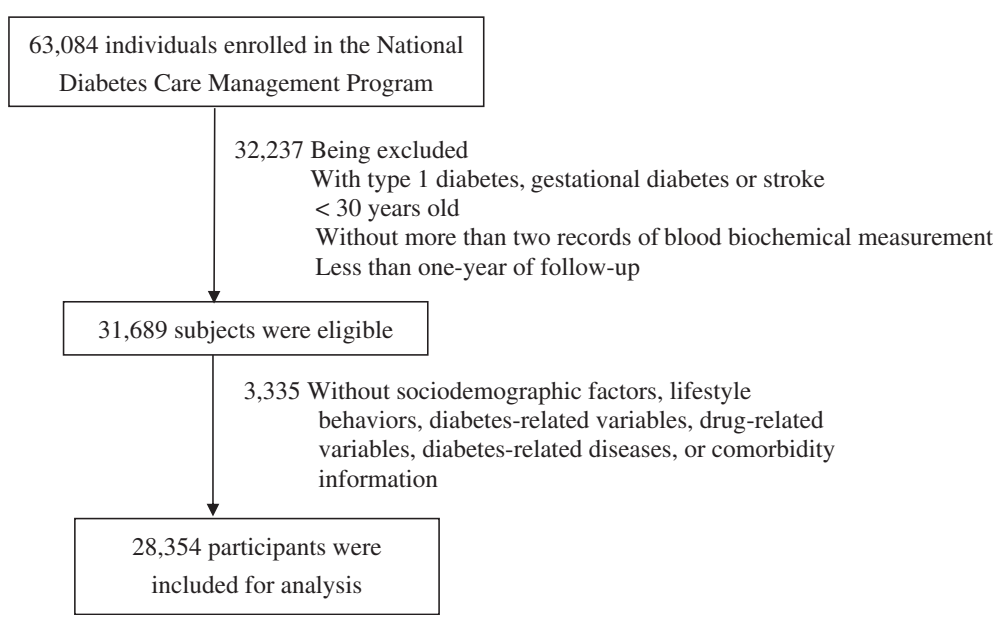

Figure 1 Flowchart of recruitment procedures for the current study. 
tests, urine tests and body measurements upon entering NDCMP. They completed standardized, computerized questionnaires administered by a case management nurse to record the previous or current status of their disease, medications and lifestyles. After a twelve-hour overnight fast, blood was drawn from an antecubital vein and sent for analysis within four hours post-collection. All patients were followed up regularly every three to six months.

\section{Outcome ascertainment}

The primary outcome measure, determined by inpatient/ outpatient claims, was based on the major ICD-9-CM discharge diagnosis code, ischemic stroke (ICD-9-CM codes 433-434). Accuracy of ischemic stroke diagnoses in NHIRD had been validated in an earlier study [18]. Accuracy of ischemic stroke diagnosis in our inpatient/outpatient claim dataset was $94 \%$, indicating NHIRD as a valid resource for population research identifying ischemic stroke. We searched NHIRD data for ischemic stroke with at least one inpatient or three outpatient claims one year after the index date, excluding incident cases within one year, to rule out cause-and-effect. Linking the unique PIN with computerized files identified 2,250 cases, followed from one year after the index date until ischemic stroke, death or withdrawal from NHI. Other chronic conditions were tabulated for 12 months prior to enrollment, using outpatient and inpatient claims data: coronary artery disease (ICD-9-CM codes 410 to 413, 414.01 to 414.05, 414.8 and 414.9), congestive heart failure (ICD-9-CM codes 428, 398.91 and 402.x1), cancer (ICD-9-CM codes 140 to 149 , 150 to 159,160 to 165,170 to 175,179 to 189,190 to 199 , 200, 202, 203, 210 to 213,215 to 229,235 to $239,654.1$, $654.10,654.11,654.12,654.13$ and 654.14), atrial fibrillation (ICD-9-CM code 427.31), hyperlipidemia (ICD-9-CM code 272), hypertension (ICD-9-CM codes 401 to 405), chronic hepatitis (ICD-9-CM codes 571, 572.2, 572.3, 572.8, 573.1, 573.2, 573.3, 573.8 and 573.9), chronic obstructive pulmonary disease (ICD-9-CM codes 490 to 496) and hypoglycemia (ICD-9-CM code 251).

\section{Statistical analysis}

The CV of FPG measurements from outpatient visits within the first year of the index date for each patient was calculated. FPG-CV was calculated only when more than two FPG measurements were performed in the first year. To adjust for the possibility that the number of visits may affect variation, the $\mathrm{CV}$ value was divided by the square root of the ratio of total visits divided by total visits minus 1 [19]. Patients were grouped into quartiles according to FPG-CV. To rule out a possible specific threshold of FPG-CV impacting the significance of the findings, sensitivity analysis classified patients into 10 subgroups according to deciles of FPG-CV. KaplanMeier cumulative incidence plots were derived. To weigh competing risk of death, an extended Cox proportional hazards model of competing risk data on stroke incidence fitted a proportional subdistribution hazards regression model with weights for subjects who underwent competing risk event of death, according to an extension of the Lunn and McNeil method [20]. Extended Cox proportional hazard models with competing risks served to evaluate the association between FPG-CV categories and incident ischemic stroke. Hazard ratios (HRs) and 95\% confidence intervals (CI) adjusted for age, gender and multiple variables. Multivariate models (1) adjusted for age (continuous) and gender; (2) additionally adjusted for tobacco (yes/no), alcohol (yes/no), duration of diabetes, type of hypoglycemic drug, antihypertensive treatment (yes/no) and obesity (body mass index $\geq 27 \mathrm{~kg} / \mathrm{m}^{2}$ ); and (3) additionally adjusted for coronary artery disease, congestive heart failure, cancer, hyperlipidemia, hypertension, atrial fibrillation, chronic hepatitis, chronic obstructive pulmonary disease and estimated glomerular filtration rate (eGFR). Interaction of FPG-CV and $\mathrm{HbA}_{1} \mathrm{c}$ was probed by adding their product terms into the full model using the likelihood ratio test for significance (set at two-tailed $P \leq 0.05)$. All analyses were performed with the SAS statistical package for Windows (Version 9.3, SAS; Cary, NC, USA).

\section{Results}

After an average 7.5 years of follow-up, there were 2,250 incident cases of ischemic stroke in type 2 diabetic patients, giving a crude incidence rate of 10.56/1,000 person-years (11.64 for men, 9.63 for women); 5,031 died, a mortality rate of $23.61 / 1,000$ person-years $(28.24$ men, 19.63 women). Table 1 shows baseline sociodemographic and clinical factors in subjects grouped according to quartiles of FPG-CV and $\mathrm{HbA}_{1} \mathrm{c}$ levels $(<7 \%$ versus $\geq 7 \%$ ). Lower FPG-CV was associated with higher mean age, lower mean duration of diabetes, triglycerides, fasting plasma glucose and $\mathrm{HbA}_{1} \mathrm{c}$, along with lower prevalence of female gender, tobacco, alcohol, three oral hypoglycemic drugs, insulin injections, insulin injections plus oral hypoglycemic drugs, congestive heart failure, cancer, hypertension and chronic obstructive pulmonary disease, and more frequent use of one or two oral hypoglycemic drugs, hypertension drug treatment, obesity and hyperlipidemia. Those with $\mathrm{HbA}_{1} \mathrm{C}<7 \%$ had a higher mean age, lower mean duration of diabetes, triglycerides, low-density lipoprotein, and fasting plasma glucose, lower prevalence of female gender, tobacco, three or more oral hypoglycemic drugs, insulin and insulin plus oral hypoglycemic drugs, and higher prevalence of no medication, one or two oral hypoglycemic drugs, hypertension drug treatment and hypertension. The Pearson correlation coefficient between baseline FPG-CV and $\mathrm{HbA}_{1} \mathrm{c}$ was weak $(r=0.225)$. Figure 1 presents the Kaplan-Meier cumulative 
Table 1 Baseline factors according to quartiles of the coefficient of variation of FPG and HbA1c levels $(n=28,354)$

\begin{tabular}{|c|c|c|c|c|c|c|c|c|}
\hline \multirow[b]{2}{*}{ Variables } & \multicolumn{4}{|l|}{ FPG-CV (\%) } & \multirow[b]{2}{*}{$P$-value } & \multicolumn{2}{|l|}{$\mathrm{HbA}_{1} \mathrm{c}(\%)$} & \multirow[b]{2}{*}{$P$-value } \\
\hline & $\begin{array}{l}\leq 14.1 \\
\text { (Number }=7,125 \text { ) }\end{array}$ & $\begin{array}{l}14.1 \text { to } 25.2 \\
\text { (Number }=7,025 \text { ) }\end{array}$ & $\begin{array}{l}25.2 \text { to } 42.0 \\
\text { (Number }=7,076 \text { ) }\end{array}$ & $\begin{array}{l}>42.0 \\
\text { (Number }=7,078 \text { ) }\end{array}$ & & $\begin{array}{l}<7.0 \\
\text { (Number }=8,091 \text { ) }\end{array}$ & $\begin{array}{l}\geq 7.0 \\
\text { (Number }=20,263 \text { ) }\end{array}$ & \\
\hline \multicolumn{9}{|l|}{ Sociodemographic factors } \\
\hline Male, number (\%) & $3,405(47.79)$ & $3,297(46.60)$ & $3,232(45.68)$ & $3,460(48.88)$ & $<0.001$ & $4,232(52.31)$ & $9,162(45.22)$ & $<0.001$ \\
\hline Age (years), mean (SD) & $60.68(11.19)$ & $60.79(11.10)$ & $60.47(11.00)$ & $59.92(11.41)$ & & $61.96(11.53)$ & $59.87(10.98)$ & $<0.001$ \\
\hline \multicolumn{9}{|l|}{ Lifestyles, number (\%) } \\
\hline Tobacco & $1,006(14.12)$ & $1,076(15.21)$ & $1,094(15.46)$ & $1,322(18.68)$ & $<0.001$ & $1,186(14.66)$ & $3,312(16.35)$ & $<0.001$ \\
\hline Alcohol & $613(8.60)$ & $652(9.22)$ & $585(8.27)$ & $708(10.00)$ & 0.002 & $777(9.60)$ & $1,781(8.79)$ & 0.03 \\
\hline \multicolumn{9}{|l|}{ Diabetes-related variables } \\
\hline Duration of diabetes (years), mean (SD) & $5.89(7.52)$ & $6.36(6.85)$ & $6.90(7.17)$ & $6.80(7.21)$ & $<0.001$ & $5.25(6.85)$ & $6.98(7.28)$ & $<0.001$ \\
\hline \multicolumn{5}{|l|}{ Type of hypoglycemic drug use, number (\%) } & $<0.001$ & & & $<0.001$ \\
\hline No medication & $127(1.78)$ & $72(1.02)$ & $61(0.86)$ & $56(0.79)$ & & $202(2.50)$ & $114(0.56)$ & \\
\hline One oral hypoglycemic drug & $1,868(26.22)$ & $1,350(19.08)$ & $980(13.85)$ & $820(11.59)$ & & 2,692 (33.27) & $2,326(11.48)$ & \\
\hline Two oral hypoglycemic drugs & $3,117(43.75)$ & $3,213(45.41)$ & $3,031(42.83)$ & $2,673(37.76)$ & & $3,768(46.57)$ & $8,266(40.79)$ & \\
\hline Three oral hypoglycemic drugs & $1,147(16.10)$ & $1,332(18.83)$ & $1,451(20.51)$ & $1,321(18.66)$ & & $873(10.79)$ & $4,378(21.61)$ & \\
\hline$>3$ oral hypoglycemic drugs & $290(4.07)$ & $364(5.14)$ & $409(5.78)$ & $416(5.88)$ & & $144(1.79)$ & $1,335(6.59)$ & \\
\hline Insulin & $93(1.31)$ & $102(1.44)$ & $202(2.85)$ & $335(4.73)$ & & $145(1.79)$ & $587(2.90)$ & \\
\hline Insulin + oral hypoglycemic drug & $483(6.78)$ & $642(9.07)$ & $942(13.31)$ & $1,457(20.58)$ & & $267(3.30)$ & $3,257(16.07)$ & \\
\hline \multicolumn{9}{|l|}{ Drug-related variables, number (\%) } \\
\hline Hypertension drug treatment & $2,626(36.86)$ & $2,600(36.75)$ & $2,637(37.27)$ & $2,447(34.57)$ & 0.004 & $3,167(39.14)$ & $7,143(35.25)$ & $<0.001$ \\
\hline \multicolumn{9}{|l|}{ Comorbidity, number (\%) } \\
\hline Obesity (BMI $\geq 27)$ & $2,688(37.73)$ & $2,640(37.31)$ & $2,635(37.24)$ & $2444(34.53)$ & $<0.001$ & $3,022(37.35)$ & $7,385(36.45)$ & 0.16 \\
\hline CAD & $533(7.48)$ & $550(7.77)$ & $558(7.89)$ & $519(7.33)$ & 0.58 & $623(7.70)$ & 1,537 (7.59) & 0.76 \\
\hline $\mathrm{CHF}$ & $138(1.94)$ & $146(2.06)$ & $155(2.19)$ & $150(2.12)$ & 0.75 & $169(2.09)$ & $420(2.07)$ & 0.97 \\
\hline Cancer & 119 (1.67) & 135 (1.91) & 138 (1.95) & $178(2.51)$ & 0.003 & $174(2.15)$ & $396(1.95)$ & 0.31 \\
\hline Hyperlipidemia & 1,894 (26.58) & $1,950(27.56)$ & $1,836(25.95)$ & $1,671(23.61)$ & $<0.001$ & $2,063(25.50)$ & $5,288(26.10)$ & 0.31 \\
\hline Hypertension & $2,969(41.67)$ & $3,056(43.19)$ & $3,165(44.73)$ & $2,878(40.66)$ & $<0.001$ & $3,669(45.35)$ & $8,399(41.45)$ & $<0.001$ \\
\hline Atrial fibrillation & $25(0.35)$ & $28(0.40)$ & $29(0.41)$ & $33(0.47)$ & 0.75 & $34(0.42)$ & $81(0.40)$ & 0.89 \\
\hline Chronic hepatitis & $706(9.91)$ & 706 (9.98) & $736(10.40)$ & 733 (10.36) & 0.68 & $832(10.28)$ & $2,049(10.11)$ & 0.68 \\
\hline COPD & 256 (3.59) & $269(3.80)$ & $307(4.34)$ & $346(4.89)$ & $<0.001$ & $366(4.52)$ & $812(4.01)$ & 0.05 \\
\hline
\end{tabular}


Table 1 Baseline factors according to quartiles of the coefficient of variation of FPG and HbA1c levels $(n=28,354)(C o n t i n u e d)$

Blood biochemical indices, mean (SD)

\begin{tabular}{|c|c|c|c|c|c|c|c|c|}
\hline Triglyceride (mg/dL) & $162.41(123.25)$ & $164.98(123.58)$ & 174.79 (136.19) & $188.96(158.34)$ & $<0.001$ & $153.12(111.28)$ & $180.61(144.54)$ & $<0.001$ \\
\hline High-density lipoprotein (mg/dL) & $46.91(14.09)$ & $46.46(13.74)$ & $46.77(14.32)$ & $46.58(14.90)$ & 0.24 & $46.51(14.35)$ & $46.75(14.23)$ & 0.21 \\
\hline Low-density lipoprotein (mg/dL) & $116.93(30.11)$ & $117.06(30.77)$ & $117.17(31.09)$ & $117.17(31.87)$ & 0.96 & $113.73(29.66)$ & $118.43(31.36)$ & $<0.001$ \\
\hline Fasting plasma glucose (mg/dL) & 157.29 (45.93) & $161.31(44.57)$ & $169.64(46.15)$ & $183.82(53.34)$ & $<0.001$ & $134.26(28.12)$ & $181.47(48.63)$ & $<0.001$ \\
\hline HbA1c (\%) & $7.55(1.52)$ & $7.78(1.48)$ & $8.13(1.51)$ & $8.51(1.58)$ & $<0.001$ & $6.30(0.51)$ & $8.67(1.32)$ & $<0.001$ \\
\hline eGFR $\left(\mathrm{mL} / \mathrm{min} / 1.73 \mathrm{~m}^{2}\right)^{\mathrm{a}}$ & $76.53(20.70)$ & $76.03(21.21)$ & $74.78(21.93)$ & $73.87(23.42)$ & $<0.001$ & $72.59(21.14)$ & $76.40(22.05)$ & $<0.001$ \\
\hline
\end{tabular}

${ }^{a}$ Missing data, number $=576$. Differences in continuous variables were tested by Student's $t$-test and ANOVA. Differences in categorical variables were tested by Chi-square test. ANOVA: analysis of variance; BMI, body

mass index; CAD: coronary artery disease; CHF: congestive heart failure; COPD: chronic obstructive pulmonary disease; CV: coefficient of variation; eGFR: estimated glomerular filtration rate; FPG: fasting plasma glucose; $\mathrm{HbA}_{1} \mathrm{C}$, glycated hemoglobin; $\mathrm{SD}$, standard deviation. 
risk for ischemic stroke within subgroups defined by FPG-CV and $\mathrm{HbA}_{1} \mathrm{c}$. Patients with FPG-CV $>14.1 \%$ faced a higher risk (log-rank test $P<0.001$, Figure $2 \mathrm{~A}$ ), as did those with $\mathrm{HbA}_{1} \mathrm{c} \geq 7.0 \%$ (log-rank test $P<0.001$, Figure 2B).

Table 2 shows HRs for ischemic stroke in subjects grouped by quartiles of FPG-CV and various $\mathrm{HbA}_{1} \mathrm{c}$ levels. Compared to patients with the first quartile, age-gender adjusted HRs in the fourth, third and second FPG-CV quartiles were 1.57 (95\% CI 1.40, 1.77), $1.44(1.27,1.62)$ and 1.24 (1.10, 1.41), respectively. Considering lifestyles, comorbidity and complications, the FPG-CV effect was slightly attenuated but still significant. Compared to patients with an $\mathrm{HbA}_{1} \mathrm{c}$ level $<7 \%$, those with an $\mathrm{HbA}_{1} \mathrm{c}$ level $7 \%$ to $8 \%, 8 \%$ to $9 \%$ and $\geq 9 \%$ manifested greater risk (agegender adjusted HR: 1.27 (1.13, 1.43), $1.55(1.37,1.75)$ and

A

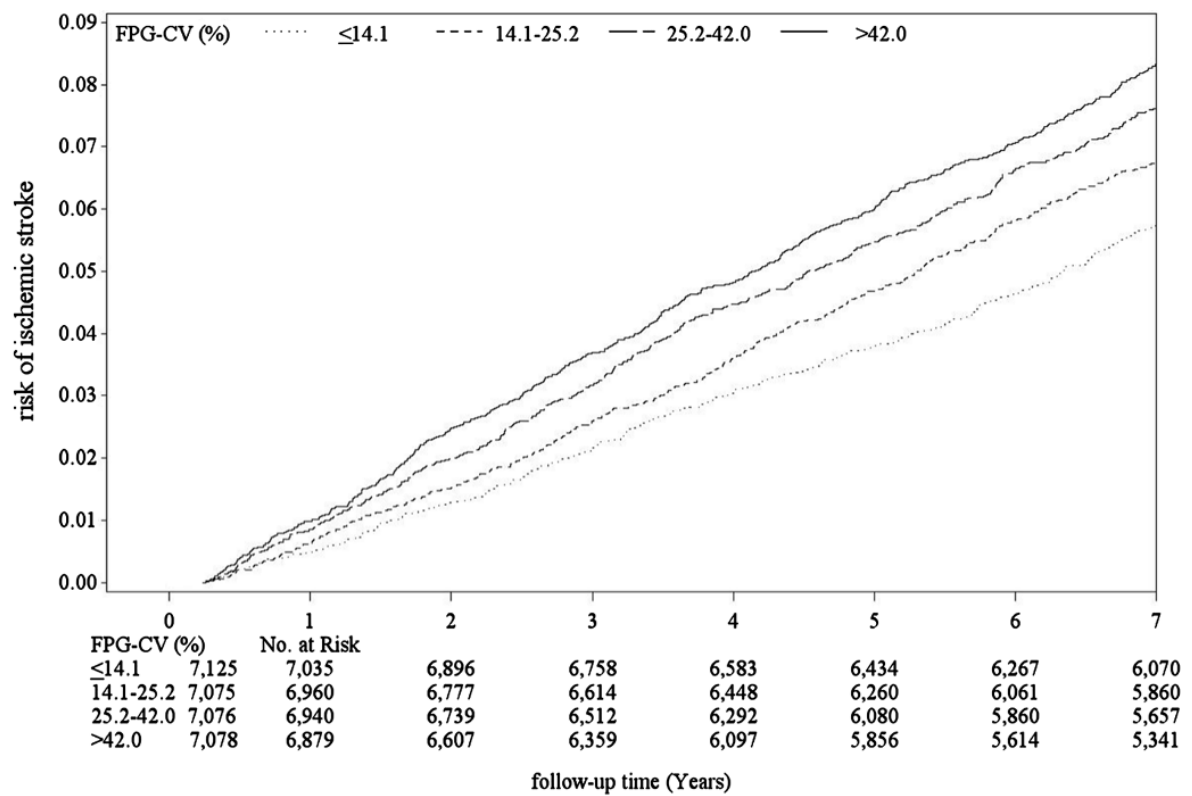

B

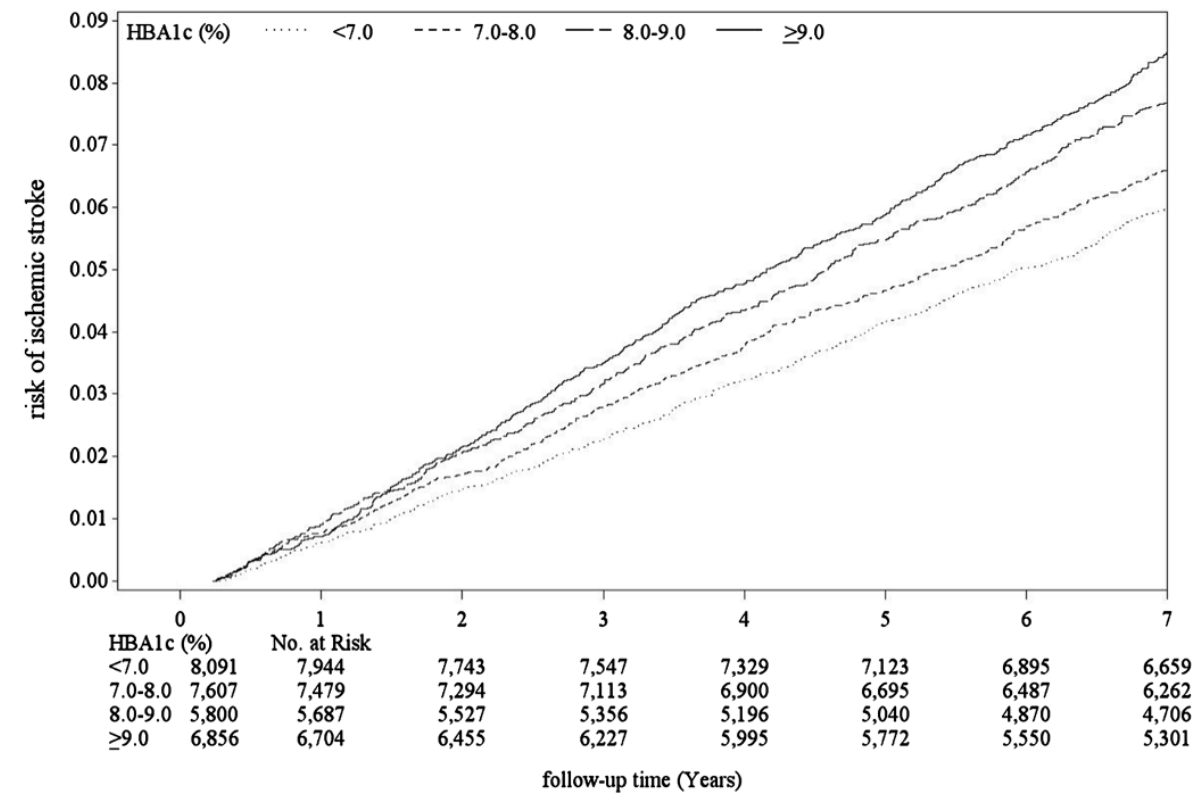

Figure 2 Risks of ischemic stroke. (A) FPG-CV and (B) $\mathrm{HbA}_{1}$ C. Log-rank test, all $p<0.001$. CV: coefficient of variation; FPG: fasting plasma: glucose; $\mathrm{HbA}_{1} \mathrm{C}$, glycated hemoglobin. 
Table 2 Hazard ratios (HRs) of ischemic stroke for quartiles of FPG-CV and $\mathrm{HbA}_{1}$ c levels $(\mathbf{n}=\mathbf{2 8 , 3 5 4 )}$

\begin{tabular}{|c|c|c|c|c|c|c|}
\hline Variables & Number & Cases & Person-years & IR & $\begin{array}{l}\text { Ischemic stroke (Number }=2,250) \\
\text { HR }(95 \% \mathrm{Cl})\end{array}$ & $P$ for trend \\
\hline \multicolumn{7}{|c|}{ Age and gender-adjusted (the first multivariate model) } \\
\hline FPG-CV (\%) & & & & & & $<0.001$ \\
\hline$\leq 14.1$ & 7,125 & 489 & $55,218.23$ & 8.86 & 1.00 & \\
\hline 14.1 to 25.2 & 7,075 & 545 & $53,865.12$ & 10.12 & $1.24(1.10,1.41)^{* * *}$ & \\
\hline 25.2 to 42.0 & 7,076 & 596 & $52,764.96$ & 11.30 & $1.44(1.27,1.62)^{* * *}$ & \\
\hline$>42.0$ & 7,078 & 620 & $51,198.11$ & 12.11 & $1.57(1.40,1.77)^{* * *}$ & \\
\hline $\mathrm{HbA}_{1} \mathrm{C}(\%)$ & & & & & & $<0.001$ \\
\hline$<7.0$ & 8,091 & 537 & $61,419.14$ & 8.74 & 1.00 & \\
\hline 7.0 to 8.0 & 7,607 & 566 & $57,820.08$ & 9.79 & $1.27(1.13,1.43)^{* * *}$ & \\
\hline 8.0 to 9.0 & 5,800 & 490 & $43,589.18$ & 11.24 & $1.55(1.37,1.75)^{* * *}$ & \\
\hline$\geq 9.0$ & 6,856 & 657 & $50,218.02$ & 13.08 & $2.06(1.85,2.31)^{* * *}$ & \\
\hline \multicolumn{7}{|c|}{ Multivariate-adjusted ${ }^{\mathrm{a}}$ (the second multivariate model) } \\
\hline FPG-CV (\%) & & & & & & $<0.001$ \\
\hline$\leq 14.1$ & 7,125 & 489 & $55,218.23$ & 8.86 & 1.00 & \\
\hline 14.1 to 25.2 & 7,075 & 545 & $53,865.12$ & 10.12 & $1.13(1.00,1.27)$ & \\
\hline 25.2 to 42.0 & 7,076 & 596 & $52,764.96$ & 11.30 & $1.24(1.10,1.40)^{* * *}$ & \\
\hline$>42.0$ & 7,078 & 620 & $51,198.11$ & 12.11 & $1.30(1.15,1.47)^{* * *}$ & \\
\hline $\mathrm{HbA}_{1} \mathrm{C}(\%)$ & & & & & & $<0.001$ \\
\hline$<7.0$ & 8,091 & 537 & $61,419.14$ & 8.74 & 1.00 & \\
\hline 7.0 to 8.0 & 7,607 & 566 & $57,820.08$ & 9.79 & $1.14(1.00,1.29)^{*}$ & \\
\hline 8.0 to 9.0 & 5,800 & 490 & $43,589.18$ & 11.24 & $1.32(1.16,1.50)^{* * *}$ & \\
\hline$\geq 9.0$ & 6,856 & 657 & $50,218.02$ & 13.08 & $1.65(1.45,1.87)^{* * *}$ & \\
\hline \multicolumn{7}{|c|}{ Multivariate-adjusted ${ }^{\mathrm{b}}$ (the third multivariate model) } \\
\hline FPG-CV (\%) & & & & & & $<0.001$ \\
\hline$\leq 14.1$ & 7,125 & 489 & $55,218.23$ & 8.86 & 1.00 & \\
\hline 14.1 to 25.2 & 7,075 & 545 & $53,865.12$ & 10.12 & $1.11(0.98,1.25)$ & \\
\hline 25.2 to 42.0 & 7,076 & 596 & $52,764.96$ & 11.30 & $1.22(1.08,1.38)^{* *}$ & \\
\hline$>42.0$ & 7,078 & 620 & $51,198.11$ & 12.11 & $1.27(1.12,1.43)^{* * *}$ & \\
\hline $\mathrm{HbA}_{1} \mathrm{C}(\%)$ & & & & & & $<0.001$ \\
\hline$<7.0$ & 8,091 & 537 & $61,419.14$ & 8.74 & 1.00 & \\
\hline 7.0 to 8.0 & 7,607 & 566 & $57,820.08$ & 9.79 & $1.14(1.01,1.29)^{*}$ & \\
\hline 8.0 to 9.0 & 5,800 & 490 & $43,589.18$ & 11.24 & $1.33(1.17,1.51)^{* * *}$ & \\
\hline$\geq 9.0$ & 6,856 & 657 & $50,218.02$ & 13.08 & $1.68(1.48,1.90)^{* * *}$ & \\
\hline FPG-CV (\%) & & & & & & 0.009 \\
\hline$\leq 14.1$ & 7,125 & 489 & $55,218.23$ & 8.86 & 1.00 & \\
\hline 14.1 to 25.2 & 7,075 & 545 & $53,865.12$ & 10.12 & $1.09(0.96,1.23)$ & \\
\hline 25.2 to 42.0 & 7,076 & 596 & $52,764.96$ & 11.30 & $1.16(1.03,1.31)^{*}$ & \\
\hline$>42.0$ & 7,078 & 620 & $51,198.11$ & 12.11 & $1.17(1.03,1.32)^{*}$ & \\
\hline $\mathrm{HbA}_{1} \mathrm{C}(\%)$ & & & & & & $<0.001$ \\
\hline$<7.0$ & 8,091 & 537 & $61,419.14$ & 8.74 & 1.00 & \\
\hline 7.0 to 8.0 & 7,607 & 566 & $57,820.08$ & 9.79 & $1.13(1.00,1.27)$ & \\
\hline
\end{tabular}


Table 2 Hazard ratios (HRs) of ischemic stroke for quartiles of FPG-CV and $\mathrm{HbA}_{\mathbf{1}} \mathrm{c}$ levels $(\mathbf{n}=\mathbf{2 8 , 3 5 4 )}(\mathrm{Continued})$

\begin{tabular}{llllll}
\hline 8.0 to 9.0 & 5,800 & 490 & $43,589.18$ & 11.24 & $1.30(1.14,1.47)^{* * *}$ \\
$\geq 9.0$ & 6,856 & 657 & $50,218.02$ & 13.08 & $1.63(1.43,1.85)^{* * *}$
\end{tabular}

${ }^{*} P<0.05 ;{ }^{* *} P<0.01 ;{ }^{* * *} P<0.001$. Multivariate-adjusted ${ }^{\text {a }}$ for age, gender, tobacco, alcohol, duration of diabetes, type of hypoglycemic drugs, hypertension drug treatment and obesity.

Multivariate-adjusted ${ }^{b}$ for coronary artery disease, congestive heart failure, cancer, hyperlipidemia, hypertension, atrial fibrillation, chronic hepatitis, chronic obstructive pulmonary disease and estimated glomerular filtration rate (eGFR) in addition to the variables in the second multivariate model. FPG-CV: coefficient of variation of fasting plasma glucose; $\mathrm{HbA}_{1} \mathrm{c}$, glycated hemoglobin; IR: incidence density rate = number of incident cases/person-years*1,000.

2.06 (1.85, 2.31), respectively). Similarly, the effect of $\mathrm{HbA}_{1} \mathrm{c}$ was slightly attenuated after multivariate adjustment. We noted linear trends across both FPG-CV and $\mathrm{HbA}_{1} \mathrm{c}$ categories. Gender-specific associations of $\mathrm{HbA}_{1} \mathrm{c}$ and FPG-CV and ischemic stroke yield similar findings. Multivariate-adjusted HRs for the third and fourth FPG-CV quartiles were $1.38(1.16,1.64)$ and 1.36 (1.14, $1.62)$, respectively, in women; $1.08(0.91,1.28)$ and 1.19 $(1.003,1.40)$, respectively, in men. Multivariate-adjusted HRs for patients with $\mathrm{HbA}_{1} \mathrm{c}$ levels $8 \%$ to $9 \%$, and $\geq 9 \%$ were $1.29(1.07,1.56)$ and $1.65(1.37,1.98)$, respectively, in women; $1.37(1.14,1.63)$ and $1.70(1.43,2.03)$, respectively, in men.

With FPG-CV and $\mathrm{HbA}_{1} \mathrm{c}$ considered simultaneously, both manifested a significant effect on ischemic stroke: multivariate-adjusted $\mathrm{HRs}$ for the fourth and third FPG-CV quartiles were $1.16(1.03,1.31)$ and $1.17(1.03$, 1.32), respectively; for $\mathrm{HbA}_{1} \mathrm{c}$ levels $8 \%$ to $9 \%$, and $\geq 9 \%$ were $1.30(1.14,1.47)$ and $1.63(1.43,1.85)$, respectively.
Sensitivity analysis assessed potential bias due to comorbidity, excluding patients with hyperglycemic hyperosmolar nonketotic coma $(\mathrm{n}=355)$, diabetic ketoacidosis $(\mathrm{n}=216)$, myocardial infarction $(\mathrm{n}=653)$, atrial fibrillation $(\mathrm{n}=115)$, hypoglycemia $(\mathrm{n}=80)$ and all comorbidity ( $\mathrm{n}=1,346)$ (Table 3). A similar significant association was found for the third and fourth FPG-CV quartiles and for $\mathrm{HbA}_{1} \mathrm{c}$ levels $7.0 \%$ to $8.0 \%, 8.0 \%$ to $9.0 \%$, and $\geq 9.0 \%$. To rule out the impact of insulin by excluding patients who use it, multivariate-adjusted HRs for the third and fourth FPG-CV quartiles were $1.22(1.08,1.38)$ and 1.29 (1.14, 1.46), respectively. With FPG-CV subgrouped based on deciles, multivariate-adjusted HRs for FPG-CV levels $20.4 \%$ to $25.2 \%, 25.2 \%$ to $30.5 \%, 30.5 \%$ to $37.6 \%, 37.6 \%$ to $47.3 \%, 47.3 \%$ to $65.3 \%$, and $>65.3 \%$ were $1.36(1.13,1.65)$, 1.35 (1.12, 1.64), 1.41 (1.17, 1.71$), 1.39$ (1.15, 1.68), 1.38 $(1.14,1.67)$ and $1.41(1.16,1.71)$, respectively. To rate the impact of potential false positives for diabetes on the findings, further analysis excluded those who were not

Table 3 Sensitivity analysis for evaluating bias due to comorbidity

\begin{tabular}{|c|c|c|c|c|c|}
\hline \multirow[b]{3}{*}{ Model $^{\mathrm{a}}$} & \multirow[b]{3}{*}{ Number } & \multicolumn{4}{|c|}{ Ischemic stroke HR $(95 \% \mathrm{Cl})$} \\
\hline & & \multicolumn{4}{|c|}{ FPG-CV (\%) } \\
\hline & & $\leq 14.1$ & 14.1 to 25.2 & 25.2 to 42.0 & $>42.0$ \\
\hline Model I & 27,999 & 1.00 & $1.10(0.97,1.24)$ & $1.21(1.08,1.37)^{* *}$ & $1.27(1.12, .144)^{* * *}$ \\
\hline Model II & 28,138 & 1.00 & $1.12(0.99,1.26)$ & $1.22(1.09,1.38)^{* *}$ & $1.27(1.13,1.44)^{* * *}$ \\
\hline Model III & 27,701 & 1.00 & $1.12(0.99,1.27)$ & $1.22(1.08,1.38)^{* *}$ & $1.27(1.12,1.44)^{* * *}$ \\
\hline Model IV & 28,239 & 1.00 & $1.11(0.98,1.26)$ & $1.22(1.08,1.37)^{* *}$ & $1.28(1.13,1.44)^{* * *}$ \\
\hline Model V & 28,274 & 1.00 & $1.11(0.98,1.26)$ & $1.22(1.08,1.38)^{* *}$ & $1.27(1.13,1.44)^{* * *}$ \\
\hline \multirow[t]{2}{*}{ Model VI } & 27,008 & 1.00 & $1.12(0.99,1.27)$ & $1.22(1.08,1.38)^{* *}$ & $1.29(1.14,1.46)^{* * *}$ \\
\hline & & \multicolumn{4}{|c|}{ HbA1c (\%) } \\
\hline Model $^{\mathrm{a}}$ & Number & $<7.0$ & 7.0 to 8.0 & 8.0 to 9.0 & $\geq 9.0$ \\
\hline Model I & 27,999 & 1.00 & $1.15(1.02,1.30)^{*}$ & $1.34(1.18,1.53)^{* * *}$ & $1.67(1.47,1.90)^{* * *}$ \\
\hline Model II & 28,138 & 1.00 & $1.14(1.01,1.28)^{*}$ & $1.33(1.17,1.51)^{* * *}$ & $1.67(1.46,1.89)^{* * *}$ \\
\hline Model III & 27,701 & 1.00 & $1.13(1.00,1.28)^{*}$ & $1.33(1.16,1.51)^{* * *}$ & $1.68(1.48,1.91)^{* * *}$ \\
\hline Model IV & 28,239 & 1.00 & $1.13(1.00,1.28)^{*}$ & $1.34(1.17,1.52)^{* * *}$ & $1.68(1.48,1.91)^{* * *}$ \\
\hline Model V & 28,274 & 1.00 & $1.15(1.02,1.30)^{*}$ & $1.34(1.18,1.52)^{* * *}$ & $1.70(1.49,1.92)^{* * *}$ \\
\hline Model VI & 27,008 & 1.00 & $1.14(1.01,1.29)^{*}$ & $1.36(1.19,1.55)^{* * *}$ & $1.69(1.48,1.93)^{* * *}$ \\
\hline
\end{tabular}

${ }^{*} \mathrm{P}<0.05$; ${ }^{*} \mathrm{P}<0.01$; ${ }^{* *} \mathrm{P}<0.001$. Multivariate-adjusted ${ }^{\mathrm{a}}$ for age, gender, tobacco, alcohol, duration of diabetes, hypoglycemic drugs, hypertension drug treatment, obesity, coronary artery disease, congestive heart failure, cancer, hyperlipidemia, hypertension, atrial fibrillation, chronic hepatitis, chronic obstructive pulmonary disease and estimated glomerular filtration rate (eGFR). Model I excluding patients with hyperglycemic hyperosmolar nonketotic coma (HHNK) (Number = 355). Model II excluding patients with diabetic ketoacidosis (DKA) (Number $=216$ ). Model III excluding patients with myocardial infarction (Number $=653)$. Model IV excluding patients with atrial fibrillation (Number = 115). Cl: confidence interval; FPG-CV: coefficient of variation of fasting plasma glucose; HR: hazard ratio; HbA1c, glycated hemoglobin. 
on medication. Multivariate-adjusted HRs for the third and fourth FPG-CV quartiles were similar (1.26 (1.12, $1.43)$ and $1.32(1.17,1.50)$, respectively). To rule out the confounding effect of $\mathrm{HbA}_{1} \mathrm{c}$, FPG-CV stratified by $\mathrm{HbA}_{1} \mathrm{c}$ $(<7.0 \%$ or $\geq 7 \%)$ was considered (Figure 3$)$. We found no significant interaction effects of FPG-CV and $\mathrm{HbA}_{1} \mathrm{c}$. Multivariate-adjusted HRs for the third and fourth FPG-CV quartiles showed significant linkage with ischemic stroke in patients with $\mathrm{HbA}_{1} \mathrm{c}<7.0 \%$; the FPG-CV fourth quartile was significantly correlated with ischemic stroke in patients with $\mathrm{HbA}_{1} \mathrm{c} \geq 7.0 \%(1.15(1.00,1.32))$.

\section{Discussion}

This study is the first to demonstrate variation in FPG measurement predicting ischemic stroke in type 2 diabetic patients more than 30 years old. Strengths of this study include a relatively large number of type 2 diabetic cases, standard data collection procedures, a sufficiently long follow-up period and available information on large numbers of potential confounding factors. Our results show that FPG-CV is a glucose variation measure that pinpoints the association between oscillating plasma glucose and ischemic stroke independent of $\mathrm{HbA}_{1} \mathrm{c}$. Our findings are relevant to the clinical management of type 2 diabetes. FPG should be measured to monitor glycemic variability as well as $\mathrm{HbA}_{1} \mathrm{c}$. Therapies now used should be evaluated for their potential to minimize glucose fluctuation and/or reduce $\mathrm{HbA}_{1} \mathrm{c}$ in type 2 diabetic patients to prevent ischemic strokes.

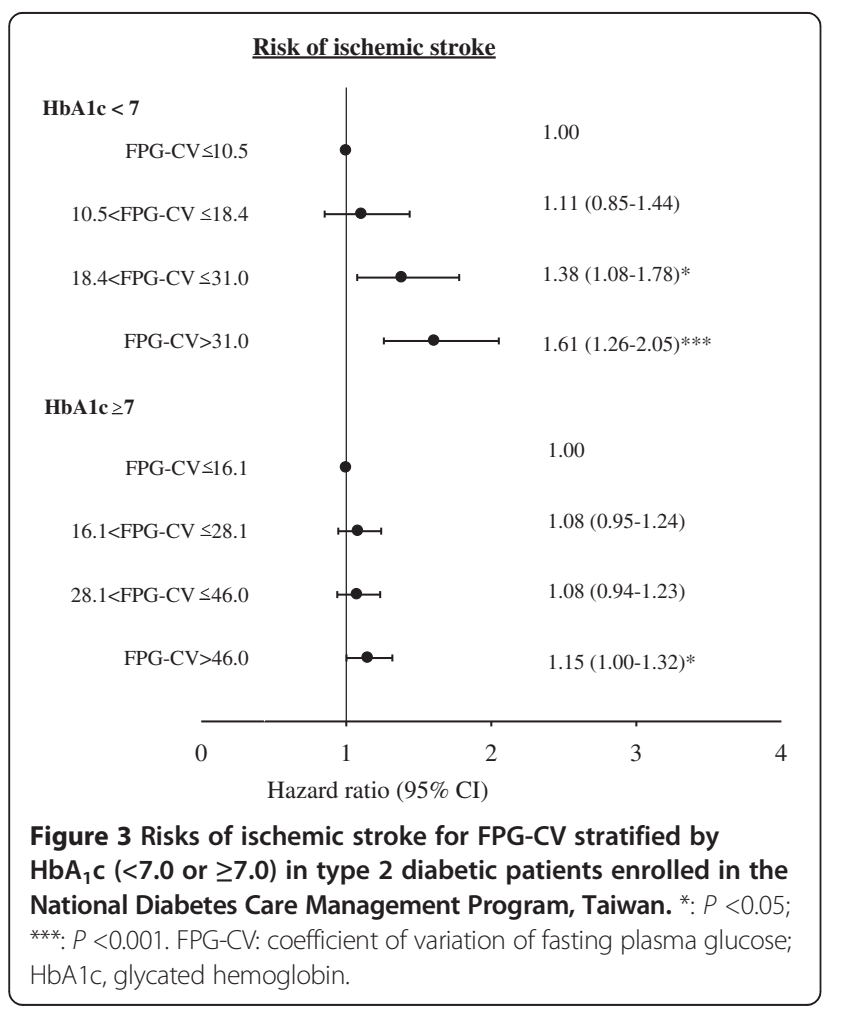

$\mathrm{HbA}_{1} \mathrm{c}$ level reflects average glucose over the preceding eight to twelve weeks of glycemic control and is viewed as a more accurate and stable measure than fasting blood glucose level [21,22]. Evidence points to elevated $\mathrm{HbA}_{1} \mathrm{c}$ as an independent risk factor for ischemic stroke $[23,24]$. However, meta-analyses and recent randomized controlled trials such as the Action to Control Cardiovascular Risk in Diabetes (ACCORD) trial, the Action in Diabetes and Vascular Disease (ADVANCE) trial, and the Veterans' Administration Diabetes Trial (VADT) found that lowering blood glucose did not appreciably reduce the pooled incidence of stroke [5,25-27]. Interpretation is complicated, partly because $\mathrm{HbA}_{1} \mathrm{c}$ is just one aspect of glycemic disorder: as an integrated measure of sustained chronic hyperglycemia, it fails to reflect glucose variability and the risks associated with extreme glucose swings [28]. Patients with similar $\mathrm{HbA}_{1} \mathrm{c}$ levels show markedly variant daily glucose excursions. Increasing evidence hints that glucose variability raises the risk of diabetic complications [8,29-31]. Consistent with previous studies, we found that $\mathrm{HbA}_{1} \mathrm{C}$ is independently associated with ischemic stroke $[19,21]$. We saw a novel predictor representing glucose instability (FPG-CV) portending greater risk of ischemic stroke. We hypothesize that the failure of therapy targeting chronic sustained hyperglycemia on ischemic stroke may be due to the fact that controlling fasting glucose and $\mathrm{HbA}_{1} \mathrm{c}$ but not glycemic variability may be inadequate. Lessened glycemic variability might improve outcome; stability of fasting plasma glucose over time should be a goal in preventing ischemic stroke. A well-designed study that entails stablizing the glucose level will verify whether glucose stability reduces ischemic stroke.

Both the Verona Diabetes and our own studies prove that FPG-CV is an independent predictor of total, cancer and cardiovascular mortality [11-15]. A strong correlation between glucose variability and mortality in critically ill patients emerged from a systemic review with at least 12 independent cohorts [32]. Stroke, like macroangiopathy, is a facet of cardiovascular disease in diabetes, poorly recognized as a specific target for evaluation.

We noted links between FPG variation and ischemic stroke, with three possible explanations. First, plausible mechanisms explain linkage of $\mathrm{HbA}_{1} \mathrm{c}$ and $\mathrm{FPG}$ variation with ischemic stroke. Oscillating plasma glucose proves more deleterious than constant high glucose on endothelial function and oxidative-stress generation, factors in macroangiopathy and, thus, predisposition to ischemic stroke [33]. Glucose fluctuations appear more relevant to atherosclerosis progression in type 2 diabetics than those with sustained hyperglycemia $[34,35]$. Recent studies indicate that change in intima-media thickness, a surrogate marker for early cerebral atherosclerosis, is 
associated with a reduction in daily glucose excursions, but not indices of chronic sustained hyperglycemia [36].

Second, oscillating plasma glucose can predispose to hypoglycemia, which may act as a precipitating factor of cerebrovascular events $[37,38]$. It has also been shown that hyperglycemia after hypoglycemia could be more dangerous than that when hypoglycemia is followed by normoglycemia [39]. In this regard, patients with welldocumented hypoglycemic episodes were more represented among subjects in the fourth quartile of FPG-CV. Yet after sensitivity analysis by excluding patients with hypoglycemia, FPG-CV showed links with incidence of ischemic stroke; this association cannot be explained by hypoglycemia in patients with high glucose variability. One could speculate FPG-CV and $\mathrm{HbA}_{1} \mathrm{c}$ as strongly interrelated, that FPG-CV seems an epiphenomenon of poor glycemic control. With no significant interactions of FPG-CV and $\mathrm{HbA}_{1} \mathrm{c}$ on ischemic stroke observed, it seems that FPG-CV and $\mathrm{HbA}_{1} \mathrm{c}$ describe separate aspects of dysglycemic impact on ischemic stroke.

Third, it is possible that glucose variation reflects the effects of coexisting conditions and incompletely quantified confounding variables that can heighten risk of ischemic stroke rather than directly cause it [13-15]. Diabetic patients, vulnerable to sugar variability, showed more comorbidity: for example, hypertension, hyperlipidemia, obesity. Baseline coexisting illness, comorbidity and complications were considered in our regression models; patients who had glucose instability with these co-factors were excluded in sensitivity analysis to disprove this possibility. Strong correlation of FPG-CV with ischemic stroke remained the same, independent of other risk factors.

This study has limitations. First, findings were limited by potential residual and unrecognized confounding variables, since this study was observational. Second, measurement errors were possible due to the large amount of data gathered from clinical practice. Third, information on subtype or size of infarction was not available. The effect of glucose variability in ischemia subtypes must be examined in future studies. Fourth, we only have one-year FPC measurements in the NDCMP dataset to estimate FPC-CV. We thus could not evaluate the effect of FPC-CV during follow-up on risk of ischemic stroke. Fifth, assessment of glucose variability is complex; quantification of glucose variability by prior studies, including FPG-CV, shows limitations $[6,7,15,40]$. No 'gold standard' currently exists to rate glucose variability. One point-counterpoint article regarding glucose variability cited $\mathrm{CV}$ and mean absolute glucose change as better markers for glucose variability [41]. Indeed, assays of fasting plasma glucose with $\mathrm{CV}$ to gauge cardiovascular risk in clinical practice are more feasible than those more complex methods, so significant to validate their utility. Future study must compare predictive capacity of glucose variance for medical outcome in diabetic cases. Finally, a growing body of evidence shows a causal relation between post-prandial hyperglycemia and cardiovascular disease independent of $\mathrm{HbA}_{1} \mathrm{c}$ and FPG [42,43]. This study did not measure post-prandial glucose and, thus, could not assess the effect of post-prandial hyperglycemia contributing to ischemic stroke. We elucidated how, in addition to $\mathrm{HbA}_{1} \mathrm{c}$, FPG-CV variation predicts ischemic stroke in type 2 diabetics. Special heed should be paid to maintain glucose concentration. Clinical trials with large sample sizes entailing intervention to stabilize glucose level should unearth evidence that glucose stability lowers the incidence of ischemic stroke.

\section{Conclusions}

Besides $\mathrm{HbA}_{1} \mathrm{c}$, FPG-CV was a potent predictor of ischemic stroke in type 2 diabetic patients, suggesting that different therapeutic strategies now in use be rated for their potential to (1) minimize glucose fluctuations and (2) reduce $\mathrm{HbA}_{1} \mathrm{c}$ level in type 2 diabetic patients to prevent ischemic stroke.

\section{Competing interests}

The authors declare that they have no competing interests.

\section{Authors' contributions}

TCL, CPY and CCL contributed equally to the design and implementation of the study: for example, field activities, quality assurance and control. CIL, CSL, $C C L$ and WYL supervised field activities. CPY, CSL, CCC, KLH and CIL helped conduct the literature review and prepared the Methods and Discussion sections. TCL and SYY designed the analytic strategy and analyzed data. All authors read and approved final manuscript.

\section{Acknowledgements}

Study was funded chiefly by the Bureau of National Health Insurance (DOH94- NH-1007), Ministry of Science and National Science Council Technology of Taiwan (NSC101-2314-B-039-017-MY3, NSC102-2314-B-039005-MY2), Health Promotion Administration, Ministry of Health \& Welfare (DOH101-HP-1102, DOH102-HP- 1102, MOHW103-HPA-H-114-133105) and Ministry of Health \& Welfare Clinical Trial and Research Center of Excellence (MOHW103-TDU-B-212- 113002).

\section{Author details}

'Department of Family Medicine, China Medical University Hospital, Taichung, Taiwan. ${ }^{2}$ Department of Medical Research, China Medical University Hospital, Taichung, Taiwan. ${ }^{3}$ School of Medicine, College of Medicine, China Medical University, Taichung, Taiwan. ${ }^{4}$ Department of Neurology, Kuang Tien General Hospital, Taichung, Taiwan. ${ }^{5}$ Department of Nutrition, Huang-Kuang University, Taichung, Taiwan. ${ }^{6}$ Graduate Institute of Integrated Medicine, College of Chinese Medicine, China Medical University, Taichung, Taiwan. ${ }^{7}$ Division of Endocrinology and Metabolism, Department of Medicine, China Medical University Hospital, Taichung, Taiwan. ${ }^{8}$ School of Chinese Medicine, College of Chinese Medicine, China Medical University, Taichung, Taiwan. ${ }^{9}$ Department of Public Health, Chung Shan Medical University, Taichung, Taiwan. ${ }^{10}$ Graduate Institute of Biostatistics, College of Management, China Medical University, Taichung, Taiwan. ${ }^{11}$ Department of Healthcare Administration, College of Medical and Health Science, Asia University, Taichung, Taiwan. 


\section{References}

1. Rautio A, Eliasson M, Stegmayr B: Favorable trends in the incidence and outcome in stroke in nondiabetic and diabetic subjects: findings from the Northern Sweden MONICA Stroke Registry in 1985 to 2003. Stroke 2008, 39:3137-3144.

2. Jeerakathil T, Johnson JA, Simpson SH, Majumdar SR: Short-term risk for stroke is doubled in persons with newly treated type 2 diabetes compared with persons without diabetes: a population-based cohort study. Stroke 2007, 38:1739-1743.

3. Goldstein LB, Bushnell CD, Adams RJ, Appel L, Braun LT, Chaturvedi S, Creager MA, Culebras A, Eckel RH, Hart RG, Hinchey JA, Howard VJ, Jauch EC, Levine SR, Meschia JF, Moore WS, Nixon JV, Pearson TA, American Heart Association Stroke Council, Council on Cardiovascular Nursing, Council on Epidemiology and Prevention, Council for High Blood Pressure Research, Council on Peripheral Vascular Disease, Interdisciplinary Council on Quality of Care and Outcomes Research: Guidelines for the primary prevention of stroke: a guideline for healthcare professionals from the American Heart Association/American Stroke Association. Stroke 2011, 42:517-584.

4. Skyler JS, Bergenstal R, Bonow RO, Buse J, Deedwania P, Gale EA, Howard BV, Kirkman MS, Kosiborod M, Reaven P, Sherwin RS, American Diabetes Association, American College of Cardiology Foundation, American Heart Association: Intensive glycemic control and the prevention of cardiovascular events: implications of the ACCORD, ADVANCE, and VA diabetes trials: a position statement of the American Diabetes Association and a scientific statement of the American College of Cardiology Foundation and the American Heart Association. Circulation 2009, 119:351-357.

5. American Diabetes Association: Standards of medical care in diabetes2010. Diabetes Care 2010, 33:S11-S61.

6. Ceriello A, Ihnat MA: 'Glycemic variability': a new therapeutic challenge in diabetes and the critical care setting. Diabet Med 2010, 27:862-867.

7. Siegelaar SE, Holleman F, Hoekstra JB, DeVries JH: Glucose variability; does it matter? Endocr Rev 2010, 31:171-182.

8. Ceriello A, Kilpatrick ES: Glycemic variability: both sides of the story. Diabetes Care 2013, 36:S272-S275.

9. Kohnert KD, Freyse EJ, Salzsieder E: Glycaemic variability and pancreatic $\beta$-cell dysfunction. Curr Diabetes Rev 2012, 8:345-354.

10. Ceriello A, Esposito K, Piconi L, Ihnat MA, Thorpe JE, Testa R, Boemi M, Giugliano D: Oscillating glucose is more deleterious to endothelial function and oxidative stress than mean glucose in normal and type 2 diabetic patients. Diabetes 2008, 57:1349-1354.

11. Muggeo M, Verlato G, Bonora E, Bressan F, Girotto S, Corbellini M, Gemma ML, Moghetti P, Zenere M, Cacciatori V, Zoppini G, De Marco R: The Verona Diabetes Study: a population-based survey on known diabetes mellitus prevalence and 5-year all-cause mortality. Diabetologia 1995, 38:318-325.

12. Muggeo M, Zoppini G, Bonora E, Brun E, Bonadonna RC, Moghetti P, Verlato G: Fasting plasma glucose variability predicts 10-year survival of type 2 diabetic patients: the verona diabetes Study. Diabetes Care 2000, 23:45-50.

13. Lin CC, Li Cl, Yang SY, Liu CS, Chen CC, Fuh MM, Chen W, Li TC: Variation of fasting plasma glucose: a predictor of mortality in patients with type 2 diabetes. Am J Med 2012, 125:e9-e18.

14. Lin CC, Li Cl, Liu CS, Lin WY, Chen CC, Yang SY, Lee CC, Li TC: Annual fasting plasma glucose variation increases risk of cancer incidence and mortality in patients with type 2 diabetes: the Taichung Diabetes Study. Endocr Relat Cancer 2012, 19:473-483.

15. Nalysnyk L, Hernandez-Medina M, Krishnarajah G: Glycaemic variability and complications in patients with diabetes mellitus: evidence from a systematic review of the literature. Diabetes Obes Metab 2010, 12:288-298.

16. Wu VC, Huang TM, Wu PC, Wang WJ, Chao CT, Yang SY, Shiao CC, Hu FC, Lai CF, Lin YF, Han YY, Chen YS, Hsu RB, Young GH, Wang SS, Tsai PR, Chen YM, Chao TT, Ko WJ, Wu KD, NSARF Group: Preoperative proteinuria is associated with long-term progression to chronic dialysis and mortality after coronary artery bypass grafting surgery. PLoS One 2012, 7:e27687.

17. National Health Insurance Administration, Ministry of Health and Welfare: The National Health Insurance Statistics, 2010; 2010. Available at: http://www.nhi.gov.tw/English/webdata/webdata.aspx? menu=11\&menu_id=296\&WD_ID=296\&webdata_id=4010; In Chinese.

18. Cheng $C L$, Kao YH, Lin SJ, Lee CH, Lai ML: Validation of the National Health Insurance Research Database with ischemic stroke cases in Taiwan. Pharmacoepidemiol Drug Saf 2011, 20:236-242.

19. Kilpatrick ES, Rigby AS, Atkin S: A1C variability and the risk of microvascular complications in type 1 diabetes: data from the Diabetes Control and Complications Trial. Diabetes Care 2008, 31:2022-2198.
20. Lunn M, McNeil D: Applying Cox regression to competing risks. Biometrics 1995, 51:524-532.

21. Goldstein DE, Little RR, Lorenz RA, Malone Jl, Nathan DM, Peterson CM, American Diabetes Association: Tests of glycemia in diabetes. Diabetes Care 2003, 26:S106-S108

22. Heo SH, Lee SH, Kim BJ, Kang BS, Yoon BW: Does glycated hemoglobin have clinical significance in ischemic stroke patients? Clin Neurol Neurosurg 2010, 112:98-102.

23. Stratton IM, Adler Al, Neil HA, Matthews DR, Manley SE, Cull CA: Association of glycaemia with macrovascular and microvascular complications of type 2 diabetes (UKPDS 35): prospective observational study. BMJ 2000, 321:405-412.

24. Selvin E, Coresh J, Shahar E, Zhang L, Steffes M, Sharrett AR: Glycaemia (haemoglobin A1c) and incident ischaemic stroke: the Atherosclerosis Risk in Communities (ARIC) Study. Lancet Neurol 2005, 4:821-826.

25. Action to Control Cardiovascular Risk in Diabetes Study Group, Gerstein HC, Miller ME, Byington RP, Goff DC Jr, Bigger JT, Buse JB, Cushman WC, Genuth S, Ismail-Beigi F, Grimm RH Jr, Probstfield JL, Simons-Morton DG, Friedewald WT: Effects of intensive glucose lowering in type 2 diabetes. N Engl J Med 2008, 358:2545-2559.

26. Duckworth W, Abraira C, Moritz T, Reda D, Emanuele N, Reaven PD, Zieve FJ, Marks J, Davis SN, Hayward R, Warren SR, Goldman S, McCarren M, Vitek ME, Henderson WG, Huang GD, VADT Investigators: Glucose control and vascular complications in veterans with type 2 diabetes. $N$ Engl J Med 2009, 360:129-139.

27. ADVANCE Collaborative Group, Patel A, MacMahon S, Chalmers J, Neal B, Billot L, Woodward M, Marre M, Cooper M, Glasziou P, Grobbee D, Hamet P, Harrap S, Heller S, Liu L, Mancia G, Mogensen CE, Pan C, Poulter N, Rodgers A, Williams B, Bompoint S, de Galan BE, Joshi R, Travert F: Intensive blood glucose control and vascular outcomes in patients with type 2 diabetes. N Engl J Med 2008, 358:2560-2572.

28. Brownlee M, Hirsch IB: Glycemic variability: a hemoglobin A1C independent risk factor for diabetic complications. JAMA 2006, 295:1707-1708.

29. Gimeno-Orna JA, Castro-Alonso FJ, Boned-Juliani B, Lou-Arnal LM: Fasting plasma glucose variability as a risk factor of retinopathy in type 2 diabetic patients. J Diabetes Complications 2003, 17:78-81.

30. Zoppini G, Verlato G, Targher G, Bonora E, Trombetta M, Muggeo M: The Verona Diabetes Study. Variability of body weight, pulse pressure and glycaemia strongly predict total mortality in elderly type 2 diabetic patients. Diabetes Metab Res Rev 2008, 24:624-628.

31. Hirsch IB, Brownlee M: Should minimal blood glucose variability become the gold standard of glycemic control? J Diabetes Complications 2005, 19:178-181.

32. Eslami S, Taherzadeh Z, Schultz MJ, Abu-Hanna A: Glucose variability measures and their effect on mortality: a systematic review. Intensive Care Med 2011, 37:583-593.

33. Monnier L, Mas E, Ginet C, Michel F, Villon L, Cristol JP, Colette C: Activation of oxidative stress by acute glucose fluctuations compared with sustained chronic hyperglycemia in patients with type 2 diabetes. JAMA 2006, 295:1681-1687.

34. Hu Y, Liu W, Huang R, Zhang X: Postchallenge plasma glucose excursions, carotid intima-media thickness, and risk factors for atherosclerosis in Chinese population with type 2 diabetes. Atherosclerosis 2010, 210:302-306.

35. Temelkova-Kurktschiev TS, Koehler C, Henkel E, Leonhardt W, Fuecker K, Hanefeld M: Postchallenge plasma glucose and glycemic spikes are more strongly associated with atherosclerosis than fasting glucose or $\mathrm{HbA} 1 \mathrm{c}$ level. Diabetes Care 2000, 23:1830-1834

36. Barbieri M, Rizzo MR, Marfella R, Boccardi V, Esposito A, Pansini A, Paolisso G: Decreased carotid atherosclerotic process by control of daily acute glucose fluctuations in diabetic patients treated by DPP-IV inhibitors. Atherosclerosis 2013, 227:349-354.

37. Zoungas S, Patel A, Chalmers J, de Galan BE, Li Q, Billot L, Woodward M, Ninomiya T, Neal B, MacMahon S, Grobbee DE, Kengne AP, Marre M, Heller S, ADVANCE Collaborative Group: Severe hypoglycemia and risks of vascular events and death. N Engl J Med 2010, 363:1410-1418.

38. Yakubovich N, Gerstein HC: Serious cardiovascular outcomes in diabetes: the role of hypoglycemia. Circulation 2011, 123:342-348.

39. Ceriello A, Novials A, Ortega E, La Sala L, Pujadas G, Testa R, Bonfigli AR, Esposito K, Giugliano D: Evidence that hyperglycemia after recovery from 
hypoglycemia worsens endothelial function and increases oxidative stress and inflammation in healthy control subjects and subjects with type 1 diabetes. Diabetes 2012, 61:2993-2997.

40. Service FJ: Glucose variability. Diabetes 2013, 62:1398-1404

41. DeVries JH: Glucose variability: where it is important and how to measure it. Diabetes 2013, 62:1405-1408.

42. DECODE Study Group, the European Diabetes Epidemiology Group: Glucose tolerance and cardiovascular mortality: comparison of fasting and 2-hour diagnostic criteria. Arch Intern Med 2001, 161:397-405.

43. Meigs JB, Nathan DM, D'Agostino RB Sr, Wilson PW, Framingham Offspring Study: Fasting and postchallenge glycemia and cardiovascular disease risk: the Framingham Offspring Study. Diabetes Care 2002, 25:1845-1850.

doi:10.1186/s12916-014-0165-7

Cite this article as: Lin et al:: Visit-to-visit variability of fasting plasma glucose as predictor of ischemic stroke: competing risk analysis in a national cohort of Taiwan Diabetes Study. BMC Medicine 2014 12:165.

\section{Submit your next manuscript to BioMed Central and take full advantage of:}

- Convenient online submission

- Thorough peer review

- No space constraints or color figure charges

- Immediate publication on acceptance

- Inclusion in PubMed, CAS, Scopus and Google Scholar

- Research which is freely available for redistribution 